\title{
Prevalence of Sarcopenia in the Korean Woman Based on the Korean National Health and Nutritional Examination Surveys
}

\author{
Hyoung-Joon Kwon', Yong-Chan $\mathrm{Ha}^{2}$, Hyoung-Moo Park ${ }^{1}$ \\ ${ }^{1}$ Department of Obstetrics and Gynecology, Chung-Ang University College of Medicine, Seoul; \\ ${ }^{2}$ Department of Orthopedic Surgery, Chung-Ang University College of Medicine, Seoul, Korea
}

\author{
Corresponding author \\ Hyoung-Moo Park \\ Department of Obstetrics and Gynecology, \\ College of Medicine, Chung-Ang University, \\ 102 Heukseok-ro, Dongjak-gu, Seoul 06973, \\ Korea \\ Tel: +82-2-6299-3172 \\ Fax: +82-2-6263-2187 \\ E-mail: hmpark52@hanmail.net
}

Received: February 7, 2016

Revised: February 23, 2016

Accepted: February 23, 2016

No potential conflict of interest relevant to this article was reported

\begin{abstract}
Background: The criteria for defining sarcopenia vary among studies and confusion has arisen when defining the cutoff value. As a result, the prevalence of sarcopenia differs markedly depending on the definition. This study used the Asian Working Group for Sarcopenia (AWGS) definition to determine the prevalence of sarcopenia among Korean women. Methods: This study was based on data obtained from the 2008 to 2011 Korean National Health and Nutrition Examination Surveys IV and V. We used the AWGS recommended cutoff value of $5.4 \mathrm{~kg} / \mathrm{m}^{2}$ to determine the prevalence of sarcopenia in Korean women. Results: The prevalence rates of sarcopenia using a cutoff value of $5.4 \mathrm{~kg} / \mathrm{m}^{2}$ were $385(19.5 \%)$ in women in their 50s, 286 (16.6\%) in women in their 60s, $293(23.7 \%)$ in women in their 70 s, and 91 (30.8\%) in women $\geq 80$ years. The prevalence rates of sarcopenia were 307 (19.0\%) in women 65 to 74 years, 194 (27.4\%) in women 75 to 84 years, and $32(40.5 \%)$ in women $\geq 85$ years. The overall prevalence among women $>50$ years was $20.2 \%$. The prevalence of sarcopenia in women $>65$ years was $22.1 \%$. Conclusions: The prevalence of sarcopenia among Korean women was within the range of values of previous research about sarcopenia. Furthermore, using $5.4 \mathrm{~kg} / \mathrm{m}^{2}$ as the cutoff value was useful to compare various studies about sarcopenia in Koreans.
\end{abstract}

Key Words: Korea, Prevalence, Sarcopenia

\section{INTRODUCTION}

In 1989, Rosenberg reported that no decline with age is more dramatic or potentially more functionally significant than the decline in lean body mass and proposed the term "sarcopenia" to describe the age-related decrease in muscle mass. [1] Since then, the implications of loss of age-related skeletal muscle mass have attracted increasing attention. These age-related changes are a major cause of impaired physical functioning in older adults and contribute to limited mobility, falls, osteoporosis, and hospitalization.[2-4] Moreover, it has now clear that skeletal muscles are also involved in endocrine and metabolic activities, such as glucose, glycogen, and lipid metabolism.[5] Therefore, sarcopenia have been widely investigated in a variety of fields.

Despite the clinical importance of sarcopenia in the elderly, the criteria for defining and clinically diagnosing sarcopenia are unclear and vary among studies

\section{Mineral Research}

This is an Open Access article distributed under the terms of the Creative Commons Attribution Non-Commercial License (http://creativecommons.org/licenses/by-nc/3.0/) which permits unrestricted non-commercial use, distribution, and reproduction in any medium, provided the origina work is properly cited.

\section{KSBMR}


and experts. Thus, several groups of experts have convened to establish consensus diagnostic criteria for sarcopenia.[6-8] Although the criteria lack clinical evidence, this is a significant advance, as many studies can now be conducted based on a single criterion without confusion. The Asian Working Group for Sarcopenia (AWGS), which includes Korea, is one of these efforts.[8]

Korea is one of the most rapidly aging countries in the world and a number of studies have focused on the prevalence, etiology, and clinical issues of sarcopenia in Korea. However, the criteria for defining sarcopenia vary among studies. Furthermore, as the distributions of muscle mass and height of Korean women in different age groups differ from those of other countries, confusion has arisen when defining cutoff values. As a result, the prevalence of sarcopenia differs markedly among studies using different definitions of sarcopenia. This study used the AWGS definition for Korean women to determine the prevalence of sarcopenia in Korean.

\section{METHODS}

This study was based on data obtained from the 2008 to 2011 Korean National Health and Nutrition Examination Surveys (KNHANES) IV and V, which is a nationally-representative survey conducted by the Korean Ministry of Health and Welfare. These surveys have been conducted periodically since 1998, using a rolling sampling design involving a complex, stratified, multistage, probability-cluster survey of a representative sample of the non-institutionalized civilian population to assess the health and nutritional status of the Korean population. Whole body dual energy $X$ ray absorptiometry (DXA) scans were performed on individual's $\geq 10$ years old from July 2008 to May 2011. A total of 5,211 women $>50$ years were included in the study. Written informed consent was given by all participants, and

Table 1. Distribution and characteristics of Korean women by age

\begin{tabular}{lcccc}
\hline Age & $\begin{array}{c}\text { Total number } \\
(\mathrm{n}=5,211)\end{array}$ & $\begin{array}{c}\text { Height } \\
(\mathrm{cm})\end{array}$ & $\begin{array}{c}\text { Weight } \\
(\mathrm{kg})\end{array}$ & $\begin{array}{c}\mathrm{SMl} \\
\left(\mathrm{kg} / \mathrm{m}^{2}\right)\end{array}$ \\
\hline $50-59$ & 1,965 & $155.7 \pm 5.2$ & $58.6 \pm 8.0$ & $5.98 \pm 0.70$ \\
$60-69$ & 1,715 & $153.4 \pm 5.2$ & $57.7 \pm 8.2$ & $6.00 \pm 0.66$ \\
$70-79$ & 1,236 & $150.0 \pm 5.5$ & $54.2 \pm 8.9$ & $5.89 \pm 0.65$ \\
$\geq 80$ & 295 & $147.0 \pm 5.4$ & $49.1 \pm 7.9$ & $5.69 \pm 0.64$ \\
\hline
\end{tabular}

SMI, skeletal mass index. the KNHANES IV and V protocols were approved by the Institutional Review Board of the Korean Center for Disease Control and Prevention.

The whole-body DXA examinations for the KNHANES study were conducted with the QDR4500A apparatus (Hologic, Bedford, MA, USA). The data included values for bone mineral content $(\mathrm{g})$, bone mineral density (BMD) $\left(\mathrm{g} / \mathrm{cm}^{2}\right)$, fat mass ( $\mathrm{g}$ ), lean mass, including bone mineral content ( $\mathrm{g}$ ), and fat percentages of the whole body and for specific anatomical regions. Appendicular skeletal muscle mass (ASM) was obtained by adding the muscle masses of the four limbs, which were calculated by assuming that all non-fat and non-bone mass is skeletal muscle.[9,10] The skeletal mass index (SMI) was defined as ASM/height ${ }^{2} .[9,10]$ The AWGS adopted a classical approach to determine the cutoff value by using the value two standard deviations below the mean for a young reference group, which was $5.4 \mathrm{~kg} / \mathrm{m}^{2}$ for women. We used $5.4 \mathrm{~kg} / \mathrm{m}^{2}$ as the cutoff value to determine prevalence for sarcopenia among Korean women.[8]

\section{RESULTS}

The 2008 to 2011 KNHANES involved 5,211 women > 50 years. The age distribution was 1,965 in their 50s, 1,715 in their 60s, 1,236 in their 70s, and $295 \geq 80$ years. Mean height and weight decreased with age. SMI increased slightly from the 50 s to $60 \mathrm{~s}$ and then decreased with age (Table 1).

The prevalence rates of sarcopenia using the $5.4 \mathrm{~kg} / \mathrm{m}^{2}$ cutoff value were 385 (19.5\%) for women in their 50s, 286 (16.6\%) for women in their 60s, 293 (23.7\%) for women in their 70 s, and 91 (30.8\%) for women $\geq 80$ years (Table 2 ). The prevalence rates of sarcopenia were 307 (19.0\%) for women 65 to 74 years, 194 (27.4\%) for women 75 to 84 years, and $32(40.5 \%)$ for women $\geq 85$ years (Table 3 ). The overall prevalence among women $>50$ years was $20.2 \%$. The prevalence of sarcopenia was $22.1 \%$ in women $>65$ years.

Table 2. Prevalence (\%) rates of sarcopenia in postmenopausal women using the $5.4 \mathrm{~kg} / \mathrm{m}^{2}$ cutoff

\begin{tabular}{lcc}
\hline Age & Number of sample & Prevalence (\%) \\
\hline $50-59$ & 1,965 & $385(19.5)$ \\
$60-69$ & 1,715 & $286(16.6)$ \\
$70-79$ & 1,236 & $293(23.7)$ \\
$\geq 80$ & 295 & $91(30.8)$ \\
Total & 5,211 & $1,055(20.2)$ \\
\hline
\end{tabular}


Table 3. Prevalence (\%) rates of sarcopenia in elderly women using the $5.4 \mathrm{~kg} / \mathrm{m}^{2}$ cutoff

\begin{tabular}{lcc}
\hline Age & Number of sample & Prevalence (\%) \\
\hline $65-74$ & 1,611 & $307(19.0)$ \\
$75-84$ & 706 & $194(27.4)$ \\
$\geq 85$ & 79 & $32(40.5)$ \\
Total & 2,396 & $531(22.1)$ \\
\hline
\end{tabular}

\section{DISCUSSION}

The definition of sarcopenia varies. Baumgartner et al.[10] suggested a height squared adjusted index, Janssen et al.[11] suggested a weight adjusted index, and Newman et al.[12] suggested a fat mass adjusted index. Furthermore, several methods have been used to determine the cutoff value, such as using the value 1 or 2 standard deviations below the mean of healthy adults or using the lowest quintile of a study population.[13,14] As a result, the prevalence of sarcopenia can vary depending on the method used. $[7,11,15]$ As interest in the endocrine role of muscle has increased, many studies have investigated the associations between sarcopenia and other clinical parameters, such as obesity and BMD.[16,17] The results of these studies differ depending on the method used.[15]

To clarify the definition of sarcopenia, various working groups have published consensus papers. Our study is the first to address the prevalence of sarcopenia using $5.4 \mathrm{~kg} /$ $\mathrm{m}^{2}$ as recommended value by the AWGS in Korea. Various studies about sarcopenia have been released in Korea.[18, 19] However, different definitions were used in each study. $[18,19]$ This phenomenon is prominent in Korea because the differences in height between age groups is greater among Koreans compared with those in other countries. [20-22] This large difference limits use of the height adjusted index. Using the height adjusted index results in a very low prevalence of sarcopenia in Korea. Thus, many studies chose to use a weight adjusted index. The prevalence of sarcopenia was $0.1 \%$ when using the height adjusted definition and $9.7 \%$ when using the weight adjusted definition in a study on the prevalence of sarcopenia using KNHNES data.[18] Another study, which was part of the Korean Sarcopenic Obesity Study, reported sarcopenia prevalence of 4.1\% using the height adjusted index and $14.2 \%$ using the weight adjusted index.[19]

Our results show that overall prevalence of sarcopenia was $20.2 \%$ in women $\geq 50$ years and $22.1 \%$ in women $\geq 65$ years. We assumed that women $>50$ years were postmenopausal and that women $>65$ years were elderly. The prevalence of sarcopenia in this study is relatively high than other studies.[23] The reason is might be related with difference of definition and characteristics of cohort.[15] Our study had several limitations. First, we only considered low muscle mass when defining sarcopenia regardless of muscle function. Second, we did not specifically exclude women with a metabolic disease, such as hyperthyroidism or diabetes mellitus, which can influence lean body mass.

Although the prevalence rates we obtained have little clinical relevance because the cutoff was not calculated using our own cohort, the value we obtained was within the range of other studies about sarcopenia.[10,12,18,24-26] Furthermore, using the $5.4 \mathrm{~kg} / \mathrm{m}^{2}$ cutoff was useful to compare with other studies about sarcopenia in Korea.

\section{REFERENCES}

1. Rosenberg IH. Summary comments. Am J Clin Nutr 1989; 50:1231-3.

2. Penninx BW, Ferrucci L, Leveille SG, et al. Lower extremity performance in nondisabled older persons as a predictor of subsequent hospitalization. J Gerontol A Biol Sci Med Sci 2000;55:M691-7.

3. Visser M, Goodpaster BH, Kritchevsky SB, et al. Muscle mass, muscle strength, and muscle fat infiltration as predictors of incident mobility limitations in well-functioning older persons. J Gerontol A Biol Sci Med Sci 2005;60:324-33.

4. Xue QL, Walston JD, Fried LP, et al. Prediction of risk of falling, physical disability, and frailty by rate of decline in grip strength: the women's health and aging study. Arch Intern Med 2011;171:1119-21.

5. Pedersen BK, Febbraio MA. Muscles, exercise and obesity: skeletal muscle as a secretory organ. Nat Rev Endocrinol 2012;8:457-65.

6. Fielding RA, Vellas B, Evans WJ, et al. Sarcopenia: an undiagnosed condition in older adults. Current consensus definition: prevalence, etiology, and consequences. International working group on sarcopenia. J Am Med Dir Assoc 2011;12:249-56.

7. Cruz-Jentoft AJ, Baeyens JP, Bauer JM, et al. Sarcopenia: European consensus on definition and diagnosis: report of the European working group on sarcopenia in older 
people. Age Ageing 2010;39:412-23.

8. Chen LK, Liu LK, Woo J, et al. Sarcopenia in Asia: consensus report of the Asian working group for sarcopenia. J Am Med Dir Assoc 2014;15:95-101.

9. Rosenberg $\mathrm{IH}$. Sarcopenia: origins and clinical relevance. J Nutr 1997;127:990s-1s.

10. Baumgartner RN, Koehler KM, Gallagher D, et al. Epidemiology of sarcopenia among the elderly in New Mexico. Am J Epidemiol 1998;147:755-63.

11. Janssen I, Heymsfield SB, Ross R. Low relative skeletal muscle mass (sarcopenia) in older persons is associated with functional impairment and physical disability. J Am Geriatr Soc 2002;50:889-96.

12. Newman AB, Kupelian V, Visser M, et al. Sarcopenia: alternative definitions and associations with lower extremity function. J Am Geriatr Soc 2003;51:1602-9.

13. de Souza Genaro P, de Medeiros Pinheiro M, Szejnfeld VL, et al. Secondary hyperparathyroidism and its relationship with sarcopenia in elderly women. Arch Gerontol Geriatr 2015;60:349-53.

14. Di Monaco M, Castiglioni C, De Toma E, et al. Presarcopenia and sarcopenia in hip-fracture women: prevalence and association with ability to function in activities of daily living. Aging Clin Exp Res 2015;27:465-72.

15. Kim TN, Choi KM. Sarcopenia: definition, epidemiology, and pathophysiology. J Bone Metab 2013;20:1-10.

16. Kim TN, Park MS, Yang SJ, et al. Body size phenotypes and low muscle mass: the Korean sarcopenic obesity study (KSOS). J Clin Endocrinol Metab 2013;98:811-7.

17. Kim JH, Choi SH, Lim S, et al. Sarcopenia and obesity: gender-different relationship with functional limitation in older persons. J Korean Med Sci 2013;28:1041-7.
18. Kim YS, Lee Y, Chung YS, et al. Prevalence of sarcopenia and sarcopenic obesity in the Korean population based on the Fourth Korean National Health and Nutritional Examination Surveys. J Gerontol A Biol Sci Med Sci 2012;67: 1107-13.

19. Kim TN, Yang SJ, Yoo HJ, et al. Prevalence of sarcopenia and sarcopenic obesity in Korean adults: the Korean sarcopenic obesity study. Int J Obes (Lond) 2009;33:885-92.

20. Cheng Q, Zhu X, Zhang X, et al. A cross-sectional study of loss of muscle mass corresponding to sarcopenia in healthy Chinese men and women: reference values, prevalence, and association with bone mass. J Bone Miner Metab 2014; 32:78-88.

21. Lim TO, Ding LM, Zaki M, et al. Distribution of body weight, height and body mass index in a national sample of Malaysian adults. Med J Malaysia 2000;55:108-28.

22. Kwon HJ, Ha YC, Park HM. The reference value of skeletal muscle mass index for defining the sarcopenia of women in Korea. J Bone Metab 2015;22:71-5.

23. Lee ES, Park HM. Prevalence of sarcopenia in healthy Korean elderly women. J Bone Metab 2015;22:191-5.

24. lannuzzi-Sucich M, Prestwood KM, Kenny AM. Prevalence of sarcopenia and predictors of skeletal muscle mass in healthy, older men and women. J Gerontol A Biol Sci Med Sci 2002;57:M772-7.

25. Landi F, Liperoti R, Fusco D, et al. Sarcopenia and mortality among older nursing home residents. J Am Med Dir Assoc 2012;13:121-6.

26. Woo J, Leung J, Sham A, et al. Defining sarcopenia in terms of risk of physical limitations: a 5-year follow-up study of 3,153 chinese men and women. J Am Geriatr Soc 2009;57: 2224-31. 